\title{
Estudio Del Liderazgo Estudiantil Desde Las Motivaciones Para Su Elección. El Caso De La Universidad De Santo Tomás De Bucaramanga (Colombia)
}

\author{
Miguel López Gómez \\ Profesor, Universidad de Santo Tomás de Bucaramanga, Colombia \\ Antonio-Manuel Rodríguez-García \\ Personal Docente Investigador, Universidad de Granada, España \\ Miriam Ágreda Montoro \\ Profesora PhD, Universidad de Jaén, España
}

doi: 10.19044/esj.2016.v12n34p370 URL:http://dx.doi.org/10.19044/esj.2016.v12n34p370

\begin{abstract}
This paper shows an investigation developed at the University of Santo Tomás (Bucaramanga) about the qualities that students consider important to practice the student leadership. For that reason, an ad hoc questionnaire with a reliability index of 0,814 has been designed. The sample consisted of 38 students (55\% male and 45\% female) from different degrees. The results show values such as charisma, commitment, consistency, fidelity, honesty, loyalty, frankness, sincerity and firmness of the person as the most important qualities that the student must have to be chosen as a representative of students.
\end{abstract}

Keywords: leadership, students, motivations, perceptions, Colombia

\section{Resumen}

En el presente artículo se muestra una investigación llevada a cabo en la Universidad de Santo Tomás (Bucaramanga) acerca de las cualidades que los estudiantes consideran importantes para ejercer como representante estudiantil, es decir, la figura de liderazgo estudiantil. Para ello, se ha diseñado un cuestionario ad hoc con un índice de fiabilidad de 0,814. La muestra ha estado compuesta por 38 estudiantes (55\% hombres y 45\% mujeres) de diversas titulaciones. Los resultados muestran valores como el carisma, el compromiso, la coherencia, la fidelidad, la honestidad, lealtad, la franqueza, la sinceridad y la firmeza de la persona como las cualidades más 
importantes que ha de tener el estudiante para ser elegido como representante de los demás.

Palabras clave: liderazgo, estudiantes, motivaciones, percepciones, Colombia

\section{Introducción}

El liderazgo estudiantil forma parte de la vida universitaria. En el momento actual cada vez más instituciones de Educación Superior están promoviendo actividades y programas que ayudan a los alumnos a desarrollar las competencias, las habilidades, actitudes y valores necesarios para ser líderes promotores del cambio social, político y transformación de la sociedad, a partir de la búsqueda de la solución de los problemas en relación con los Derechos Humanos y la armonía que debe existir entre hombrenaturaleza-sociedad y cultura (Cáceres, 2007; Cáceres, Trujillo, Hinojo, Aznar y García, 2012; Cáceres, Sachicola e Hinojo, 2015). Cátedras de liderazgo, seminarios, talleres, cursos formales, conferencias... son impartidas en las universidades con el fin de profundizar en este tema que se considera hoy vital y, por tanto, se constata como un gran desafío en el siglo XXI, y es la formación del profesional como un auténtico líder.

\section{Liderazgo: breve conceptualización}

La atención sobre el estudio del liderazgo en el campo científico ha estado enfocada desde múltiples ópticas (Jaime, Cáceres e Hinojo, 2016). Algunos investigadores se han centrado en los rasgos de la personalidad, rasgos físicos o comportamientos del líder, otros han estudiado cómo los aspectos de la situación afectan la actuación de los líderes. Hay quienes han extendido sus puntos de vista anteriores al extremo de sugerir que no existe nada como el liderazgo, sostienen que son falsos los éxitos y fracasos organizacionales con frecuencia atribuidos al líder, la situación tiene un impacto mayor en cómo funciona la organización que cualquier individuo incluyendo el líder. (Meinde \& Ehrlich, 1987).

En este contexto, existen múltiples concepciones sobre liderazgo según distintos autores. En la siguiente tabla presentamos algunas de las más importantes:

Tabla 1 Conceptos de liderazgo

\begin{tabular}{|c|c|}
\hline Autor & Concepto \\
\hline Bennis (1959) & $\begin{array}{l}\text { Proceso mediante el cual un agente induce a } \\
\text { un subordinado a comportarse de una } \\
\text { manera deseada. }\end{array}$ \\
\hline Fiedler (1967) & $\begin{array}{l}\text { Dirigir y coordinar el trabajo de los } \\
\text { miembros del grupo. }\end{array}$ \\
\hline Merton (1969) & Relación interpersonal en la que otros \\
\hline
\end{tabular}




\begin{tabular}{|l|l|}
\hline $\begin{array}{l}\text { Bass (1985) } \\
\text { Tichy y Devanna, (1986) }\end{array}$ & $\begin{array}{l}\text { cumplen porque quieren, no porque tengan } \\
\text { que hacerlo. } \\
\text { Transformar a los seguidores, al crear } \\
\text { visiones de las metas que puedan alcanzarse } \\
\text { y articular para los seguidores las formas de } \\
\text { alcanzar esas metas. }\end{array}$ \\
\hline Rauch y Behling (1984) & $\begin{array}{l}\text { Proceso de influir a un grupo organizado } \\
\text { hacia el cumplimiento de unas metas. }\end{array}$ \\
\hline Campbell (1991) & $\begin{array}{l}\text { Acciones que enfocan recursos para crear } \\
\text { oportunidades deseables. }\end{array}$ \\
\hline Hogan, Curply y Hogan (1994) & $\begin{array}{l}\text { El trabajo del líder es crear condiciones para } \\
\text { que el equipo sea efectivo. }\end{array}$ \\
\hline $\begin{array}{l}\text { Los fines del liderazgo incluyen obtener } \\
\text { resultados a través de otros, y los medios del } \\
\text { liderazgo incluyen la capacidad de construir } \\
\text { equipos cohesivos y orientados a las metas, } \\
\text { los buenos líderes son los que construyen } \\
\text { equipos para obtener resultados entre una } \\
\text { variedad de situaciones }\end{array}$ \\
\hline
\end{tabular}

Fuente: Elaboración propia

Las definiciones de liderazgo difieren en muchas formas y se debe a que los investigadores exploran distintos aspectos del liderazgo. Una definición de liderazgo puede influir a quien es considerado un líder apropiado para el estudio. No existe una definición correcta para el liderazgo. De ahí que el liderazgo tenga algunas implicaciones como, por ejemplo, que el liderazgo es tanto una ciencia como un arte. El ámbito de la ciencia del liderazgo se refleja en la cantidad de estudios aproximadamente 8.000 según Bass, (1990). No se puede concluir que ser un experto investigador en liderazgo sea una condición para ser un buen líder. Algunos pueden ser líderes exitosos sin haber recibido capacitación. El estudio no es un requisito previo para el ejercicio del liderazgo, pero si ayuda a orientar algunas situaciones. El liderazgo requiere habilidades para superar y enfrentar las situaciones y aquí se convierte en un arte.

Tal y como señala Vázquez (2010:121), el liderazgo es la habilidad directiva que dentro del ámbito empresarial se considera hoy la más importante. El liderazgo es fácilmente reconocible en cualquier entorno. Por ejemplo, en términos deportivos, comentamos que un determinado jugador de un equipo es un líder.

\subsection{Tipos de liderazgo}

Atendiendo a la clasificación aportada por Vázquez (2010, p.128), encontraríamos seis tipos de líderes con sus propias características:

- Líderes coercitivos: este tipo de liderazgo se caracteriza por demandar de sus colaboradores el acatamiento inmediato de las 
órdenes que impone. Son líderes orientados al logro y con un buen auto-control. Este tipo de liderazgo obtiene sus mejores frutos en situaciones de crisis, cuando se trata de reestructurar o de tratar con empleados problemáticos, generalmente tiene un impacto negativo en las relaciones interpersonales de la organización y en el clima laboral.

- Líderes autoritarios: Es un liderazgo muy útil cuando se trata de movilizar a la gente hacia una nueva dirección. Estos líderes tienen buenas habilidades para promover los cambios, son muy empáticos. El líder autoritario es visionario, no afecta el clima organizacional de la empresa o Institución educativa.

- Líderes afiliativos: Fundamentan su comportamiento en la persona. Para ellos lo más importantes es crear afiliativos dentro de la organización. Es un tipo de liderazgo muy afectivo en las organizaciones en casos de problemas que afecten el buen ambiente de la organización y las relaciones interpersonales. Es un buen liderazgo para motivar a la gente en caso de estrés.

- Líderes democráticos: Fomentan la comunicación dentro de las organizaciones. Escuchan las aspiraciones de los demás. Lideran equipos en forma excelente y son hábiles para crear consensos y para estimular el liderazgo organizacional. Refuerzan un buen clima en las organizaciones empresariales y educativas.

- Líderes marca-pasos: Posibilitan conseguir estándares de rendimiento muy altos en las organizaciones. Fundamentan su comportamiento en que el colaborador haga lo que el líder hace. Este estilo de liderazgo es necesario para obtener resultados de un equipo en poco tiempo. A veces su impacto en el clima organizacional es negativo.

- Líderes coaching: Liderazgo muy útil para desarrollar a la gente de cara al futuro. Con su empatía contribuyen a la formación de los demás y ayudan a desarrollar fortalezas para sacar adelante los proyectos. Son positivos para el clima organizacional.

\subsection{Cualidades del líder}

En el marco de este tema de actualidad Lorenzo (2005), nos da algunas de sus aportaciones en sus estudios monográficos. El liderazgo es una función de construcción de las organizaciones. Aquí consideramos a las escuelas, a los colegios y universidades como organizaciones con vida propia, que nacen y crecen con el tiempo, hasta su decrepitud. En este sentido se entienden en permanente construcción, se van haciendo con el tiempo, tienen su propia historia. El liderazgo le da la impronta o el carácter a cada etapa histórica de la Institución educativa. El liderazgo se constituye 
en el motor dinamizador de la construcción histórica, social y cultural del centro educativo. Existen una gran cantidad de variables sobre las concepciones del liderazgo en las organizaciones y que buscan responder a la pregunta: ¿Qué se entiende por liderazgo en una organización? Hay respuestas centradas en las cualidades del individuo para explicar el liderazgo. El líder es la persona que posee unas cualidades que los demás no tienen en alguna dimensión humana. Gento (1996, p.186) elabora un cuadro inspirándose en las características de los líderes.

Tabla 2 Características de Líderes según Gento (1996)

\begin{tabular}{|c|c|c|}
\hline $\begin{array}{c}\text { RASGOS } \\
\text { PERSONALES }\end{array}$ & $\begin{array}{c}\text { VALORES } \\
\text { ENCARNADOS }\end{array}$ & $\begin{array}{c}\text { HÁBITOS DE } \\
\text { COMPORTAMIENTO }\end{array}$ \\
\hline $\begin{array}{l}\text { 1.- Atracción personal } \\
\text { 2.- Autenticidad } \\
\text { 3.- Simpatía } \\
\text { 4.- Poder convincente } \\
\text { 5.- Poder estimulante }\end{array}$ & $\begin{array}{l}\text { 1. Integridad } \\
\text { 2. Madurez } \\
\text { 3. Amplitud de miras } \\
\text { 4. Cooperación solidaria } \\
\text { 5. Generosidad }\end{array}$ & $\begin{array}{l}\text { Dirigir eficazmente } \\
\text { Distribuir a las personas } \\
\text { Decidir en la acción } \\
\text { Planificar constantemente } \\
\text { Esforzarse por encima del pago } \\
\text { Controlar los detalles } \\
\text { Motivar a los colaboradores } \\
\text { Trabajar sin renuncia } \\
\text { Formar y entrenar } \\
\text { Tratar individualizadamente }\end{array}$ \\
\hline
\end{tabular}

\section{Liderazgo estudiantil}

La universidad no es solo un entorno para la formación en todos los aspectos de la persona, sino además es un observatorio privilegiado de la realidad. El estudiante líder debe ser capaz de ver más allá de lo evidente, analizar las causas y consecuencias de lo que le rodea y capacitarse para comprometerse con la transformación de su entorno (Cáceres, Lorenzo y Sola, 2008). Consideramos que todas las acciones y los esfuerzos de los estudiantes universitarios son realmente fructíferos y exitosos si van acompañados de una capacitación adecuada y de la experiencia personal y vital.

La voluntad necesita ayuda en el camino para mantenerse en la firmeza de los ideales, de los sueños, de las grandes metas y logros transformadores de la historia, de otra manera se pueden perder por las acciones, gestiones y preocupaciones de la vida cotidiana, que a veces se pueden convertir en el fin último del quehacer de la vida universitaria (Cáceres, 2007; Cáceres, Trujillo, Hinojo, Aznar y García, 2012).

El liderazgo se debe ver como un compromiso de superación personal, desarrollo de las potencialidades humanas, como una actitud de servicio. La actitud de quien es consciente de la realidad que le rodea, es capaz de poner al servicio de los demás lo mejor de sí mismo, con una actitud de responsabilidad social. El verdadero líder se compromete primero con su 
propia formación en conocimientos y capacidades, para ser capaz de ofrecer a los demás lo mejor de sí mismo en un ejercicio de coherencia.

En toda institución educativa, especialmente en las universidades, existen estudiantes que son elegidos democráticamente por sus compañeros, como representantes de curso, representantes ante la facultad, ante el consejo académico y a otros cargos similares la idea que tienen los docentes es que estos influyen en la toma de decisiones de los grupos.

Es importante que en toda Universidad exista una dirección de asuntos estudiantiles, que dedique parte de su tiempo a la investigación para observar y analizar quiénes son los líderes estudiantiles y determinar a tiempo si influyen positivamente en sus compañeros, en caso contrario reorientarlos, e invitarlos a seminarios de formación en el liderazgo.

En la Universidad se debe fomentar y alentar actitudes de liderazgo, en bien de la ciencia, la investigación, la tecnología, la cultura, el cambio, la transformación social y política. etc. Dentro de un marco de libertad de pensamiento y expresión. Con estímulos para estudiantes que lideren proyectos en lo social y lo político.

Desde la cátedra los Docentes deben ser formadores de líderes estudiantiles en lo social y lo político, mediante la contextualización de los programas académicos, fomentando el trabajo en equipo, asumiendo el rol de maestros líderes, reclamando los derechos y siendo éticos en el ejercicio de la profesión. El liderazgo hace parte de la competencia como Docentes.

\section{Método}

La investigación se ha llevado a cabo en la Universidad de Santo Tomás (Bucaramanga, Colombia), con el objetivo de conocer qué cualidades ha de disponer un estudiante para ser elegido como líder, esto es, como representante estudiantil. Para ello, se ha seguido una metodología de corte cuantitativo, centrándonos en una investigación descriptiva, empleando un cuestionario como estrategia de información construido ad hoc (López, 2016), validado mediante juicio de expertos y con un índice de fiabilidad calculado a través del alfa de cronbach con valor de 0,814.

La muestra ha estado compuesta por un total de 38 estudiantes, cuyas edades se encuentran en mayor medida en torno a los 19 y los 25 años (78,9\%). En cuanto al sexo podemos apreciar en la muestra participante una relación equilibrada, un porcentaje de hombres del 55,3\% y de mujeres un 44,7\%. Siendo algo mayor el de hombres como lo demuestra la tabla.

La muestra participante son estudiantes de las distintas facultades de la Universidad Santo Tomás de Bucaramanga. Los porcentajes más altos son estudiantes de Derecho 21\%, Odontología 13.2\% Negocios Internacionales 10.5\%, Economía 10\%, se tuvo en cuenta el número de estudiantes de cada facultad. Siendo la más numerosa la de la Facultad de Derecho. 
Tabla 3 Titulación a la que pertenecen

\begin{tabular}{|c|r|r|}
\hline & Frecuencia & Porcentaje \\
\hline ING.TELECOMUNICACIONES & 2 & 5,3 \\
\hline ADMINISTRACIÓN AGROPECUARIA & 2 & 5,3 \\
\hline QUÍMICA AMBIENTAL & 3 & 7,9 \\
\hline DERECHO & 8 & 21,1 \\
\hline NEGOCIOS INTERNACIONALES & 4 & 10,5 \\
\hline LABORATORIO DENTAL & 3 & 7,9 \\
\hline ODONTOLOGÍA & 5 & 13,2 \\
\hline ECONOMÍA & 4 & 10,5 \\
\hline ING. MECATRÓNICA & 2 & 5,3 \\
\hline ING. INDUSTRIAL & 2 & 5,3 \\
\hline CONTADURÍA PÚBLICA & 2 & 5,3 \\
\hline CULTURA FÍSICA, RECREACIÓN Y & 1 & 2,6 \\
\hline DEPORTES & & 100,0 \\
\hline
\end{tabular}

\section{Resultados}

A continuación, se presentan los datos más relevantes acerca de las motivaciones que tienen en cuenta los estudiantes para la elección de los representantes estudiantiles.

Tabla 4 Estadísticos descriptivos: Cualidades del líder

\begin{tabular}{|c|c|c|}
\hline Cualidades & Media & $\begin{array}{c}\text { Desv } \\
\text { típ. }\end{array}$ \\
\hline Ser un excelente estudiante .buenas calificaciones & 3,24 &, 820 \\
\hline El carisma & $\mathbf{3 , 5 8}$ &, 552 \\
\hline La coherencia & $\mathbf{3 , 7 6}$ &, 431 \\
\hline La fidelidad & $\mathbf{3 , 9 2}$ &, 273 \\
\hline La honestidad & $\mathbf{3 , 6 8}$ &, 620 \\
\hline La lealtad & $\mathbf{3 , 9 2}$ &, 273 \\
\hline La inteligencia interpersonal, habilidades sociales y & $\mathbf{3 , 8 4}$ &, 370 \\
\hline La experiencia en cargos de representación estudiantil & 3,39 &, 638 \\
\hline La presentación personal & & \\
\hline El género & 2,95 &, 899 \\
\hline La simpatía & 3,18 &, 766 \\
\hline La humildad & 2,16 & 1,053 \\
\hline La sencillez & 3,29 &, 835 \\
\hline La franqueza & 3,45 &, 760 \\
\hline La sinceridad & 3,47 &, 725 \\
\hline La firmeza & 2,97 &, 753 \\
\hline La decisión & $\mathbf{3 , 6 1}$ &, 547 \\
\hline La sensibilidad social, la cercanía con las problemáticas del & $\mathbf{3 , 7 1}$ &, 460 \\
\hline contexto político, influyen en la elección & $\mathbf{3 , 6 8}$ &, 620 \\
\hline & $\mathbf{3 , 7 4}$ &, 554 \\
\hline & 3,24 &, 820 \\
\hline
\end{tabular}


En la tabla 4 podemos observar las cualidades que los estudiantes consideran más importantes para ser escogido como líder. De este modo, encontramos que las cualidades que mayor se valoran para elegir a un líder serían: el carisma, la coherencia, fidelidad, honestidad, lealtad, franqueza, sinceridad, firmeza, decisión y la personalidad fuerte. Por el contrario, las menos importantes serían la experiencia en cargos de gestión estudiantil y el género. De una manera más detallada, en la tabla 5 se presentan una tabla de frecuencias y porcentajes, así como la distribución de las respuestas en función del grado de acuerdo de manera pormenorizada.

Tabla 5 Frecuencias y porcentajes sobre las motivaciones para la elección del líder estudiantil

\begin{tabular}{|c|c|c|c|}
\hline Ítem & $\begin{array}{l}\text { Grado de } \\
\text { acuerdo }\end{array}$ & Frecuencia & Porcentaje \\
\hline \multirow{3}{*}{$\begin{array}{l}\text { El carisma es la cualidad fundamental del } \\
\text { líder estudiantil para persuadir y movilizar a } \\
\text { las personas a realizar sus proyectos }\end{array}$} & Poco acuerdo & 1 & 2,6 \\
\hline & De acuerdo & 14 & 36,8 \\
\hline & $\begin{array}{l}\text { Totalmente de } \\
\quad \text { acuerdo }\end{array}$ & 23 & 60,5 \\
\hline \multirow{2}{*}{$\begin{array}{l}\text { La coherencia es un valor personal que debe } \\
\text { reunir un líder estudiantil }\end{array}$} & De acuerdo & 9 & 23,7 \\
\hline & $\begin{array}{l}\text { Totalmente de } \\
\text { acuerdo }\end{array}$ & 29 & 76,3 \\
\hline \multirow{2}{*}{$\begin{array}{l}\text { El compromiso es un valor personal que debe } \\
\text { reunir un líder estudiantil }\end{array}$} & De acuerdo & 3 & 7,9 \\
\hline & $\begin{array}{l}\text { Totalmente de } \\
\text { acuerdo }\end{array}$ & 35 & 92,1 \\
\hline \multirow{3}{*}{$\begin{array}{l}\text { La fidelidad es un valor personal que debe } \\
\text { reunir un líder estudiantil }\end{array}$} & Poco acuerdo & 3 & 7,9 \\
\hline & De acuerdo & 6 & 15,8 \\
\hline & $\begin{array}{l}\text { Totalmente de } \\
\text { acuerdo }\end{array}$ & 28 & 76,3 \\
\hline \multirow{2}{*}{$\begin{array}{l}\text { La honestidad es un valor personal que debe } \\
\text { reunir un líder estudiantil }\end{array}$} & De acuerdo & 3 & 7,9 \\
\hline & $\begin{array}{l}\text { Totalmente de } \\
\text { acuerdo }\end{array}$ & 35 & 92,1 \\
\hline \multirow{2}{*}{$\begin{array}{l}\text { La lealtad es un valor personal que debe } \\
\text { reunir un líder estudiantil }\end{array}$} & De acuerdo & 6 & 15,8 \\
\hline & $\begin{array}{l}\text { Totalmente de } \\
\text { acuerdo }\end{array}$ & 32 & 84,2 \\
\hline \multirow{3}{*}{$\begin{array}{l}\text { Para ser elegido es necesario tener } \\
\text { desarrollada la inteligencia interpersonal, } \\
\text { habilidades sociales y políticas }\end{array}$} & Poco acuerdo & 3 & 7,9 \\
\hline & De acuerdo & 17 & 44,7 \\
\hline & $\begin{array}{l}\text { Totalmente de } \\
\quad \text { acuerdo }\end{array}$ & 18 & 47,4 \\
\hline \multirow{4}{*}{$\begin{array}{l}\text { La experiencia en cargos de representación } \\
\text { estudiantil y en liderazgo social político son } \\
\text { motivos para la elección }\end{array}$} & $\begin{array}{l}\text { Totalmente en } \\
\text { desacuerdo }\end{array}$ & 3 & 7,9 \\
\hline & Poco acuerdo & 7 & 18,4 \\
\hline & De acuerdo & 17 & 44,7 \\
\hline & $\begin{array}{l}\text { Totalmente de } \\
\text { acuerdo }\end{array}$ & 11 & 28,9 \\
\hline \multirow{2}{*}{$\begin{array}{l}\text { La presentación personal es característica } \\
\text { esencial y decisiva para la elección como } \\
\text { representante estudiantil }\end{array}$} & Poco acuerdo & 8 & 21,1 \\
\hline & De acuerdo & 15 & 39,5 \\
\hline
\end{tabular}




\begin{tabular}{|c|c|c|c|}
\hline & $\begin{array}{l}\text { Totalmente de } \\
\text { acuerdo }\end{array}$ & 15 & 39,5 \\
\hline \multirow[t]{4}{*}{$\begin{array}{l}\text { El género es característica esencial y decisiva } \\
\text { para la elección como representante estudiantil }\end{array}$} & $\begin{array}{l}\text { Totalmente en } \\
\text { desacuerdo }\end{array}$ & 12 & 31,6 \\
\hline & Poco acuerdo & 14 & 36,8 \\
\hline & De acuerdo & 6 & 15,8 \\
\hline & $\begin{array}{l}\text { Totalmente de } \\
\text { acuerdo }\end{array}$ & 6 & 15,8 \\
\hline \multirow{4}{*}{$\begin{array}{l}\text { La simpatía es característica esencial y } \\
\text { decisiva para la elección como representante } \\
\text { estudiantil }\end{array}$} & $\begin{array}{l}\text { Totalmente en } \\
\text { desacuerdo }\end{array}$ & 1 & 2,6 \\
\hline & Poco acuerdo & 6 & 15,8 \\
\hline & De acuerdo & 12 & 31,6 \\
\hline & $\begin{array}{l}\text { Totalmente de } \\
\text { acuerdo }\end{array}$ & 19 & 50,0 \\
\hline \multirow{3}{*}{$\begin{array}{l}\text { La humildad es característica esencial y } \\
\text { decisiva para la elección como representante } \\
\text { estudiantil }\end{array}$} & Poco acuerdo & 6 & 15,8 \\
\hline & De acuerdo & 9 & 23,7 \\
\hline & $\begin{array}{l}\text { Totalmente de } \\
\text { acuerdo }\end{array}$ & 23 & 60,5 \\
\hline \multirow{3}{*}{$\begin{array}{l}\text { La sencillez es característica esencial y decisiva } \\
\text { para la elección como representante estudiantil }\end{array}$} & Poco acuerdo & 5 & 13,2 \\
\hline & De acuerdo & 10 & 26,3 \\
\hline & $\begin{array}{l}\text { Totalmente de } \\
\text { acuerdo }\end{array}$ & 23 & 60,5 \\
\hline \multirow[t]{4}{*}{$\begin{array}{l}\text { La presentación de la candidatura ha sido } \\
\text { organizada por los profesores y directivos }\end{array}$} & $\begin{array}{l}\text { Totalmente en } \\
\text { desacuerdo }\end{array}$ & 9 & 23,7 \\
\hline & Poco acuerdo & 16 & 42,1 \\
\hline & De acuerdo & 4 & 10,5 \\
\hline & $\begin{array}{l}\text { Totalmente de } \\
\quad \text { acuerdo }\end{array}$ & 9 & 23,7 \\
\hline \multirow[t]{4}{*}{$\begin{array}{l}\text { La personalidad fuerte es un rasgo de la } \\
\text { personalidad valorado en la elección }\end{array}$} & $\begin{array}{l}\text { Totalmente en } \\
\text { desacuerdo }\end{array}$ & 2 & 5,3 \\
\hline & Poco acuerdo & 5 & 13,2 \\
\hline & De acuerdo & 23 & 60,5 \\
\hline & $\begin{array}{l}\text { Totalmente de } \\
\text { acuerdo }\end{array}$ & 8 & 21,1 \\
\hline \multirow{3}{*}{$\begin{array}{l}\text { La franqueza es un rasgo de la personalidad } \\
\text { valorado en la elección }\end{array}$} & Poco acuerdo & 1 & 2,6 \\
\hline & De acuerdo & 13 & 34,2 \\
\hline & $\begin{array}{l}\text { Totalmente de } \\
\text { acuerdo }\end{array}$ & 24 & 63,2 \\
\hline \multirow{2}{*}{$\begin{array}{l}\text { La sinceridad es un rasgo de la personalidad } \\
\text { valorado en la elección }\end{array}$} & De acuerdo & 11 & 28,9 \\
\hline & $\begin{array}{l}\text { Totalmente de } \\
\text { acuerdo }\end{array}$ & 27 & 71,1 \\
\hline \multirow[t]{3}{*}{$\begin{array}{l}\text { La firmeza es un rasgo de la personalidad } \\
\text { valorado en la elección }\end{array}$} & $\begin{array}{l}\text { Totalmente en } \\
\text { desacuerdo }\end{array}$ & 1 & 2,6 \\
\hline & De acuerdo & 9 & 23,7 \\
\hline & $\begin{array}{l}\text { Totalmente de } \\
\text { acuerdo }\end{array}$ & 28 & 73,7 \\
\hline \multirow{2}{*}{$\begin{array}{l}\text { Es la decisión un rasgo de la personalidad } \\
\text { valorado en la elección }\end{array}$} & Poco acuerdo & 2 & 5,3 \\
\hline & De acuerdo & 6 & 15,8 \\
\hline
\end{tabular}




\begin{tabular}{|l|c|c|c|}
\hline & $\begin{array}{c}\text { Totalmente de } \\
\text { acuerdo }\end{array}$ & 30 & 78,9 \\
\hline $\begin{array}{l}\text { La personalidad fuerte, la franqueza, la } \\
\text { sinceridad, la decisión firme son valorados en } \\
\text { la elección }\end{array}$ & $\begin{array}{c}\text { Totalmente en } \\
\text { desacuerdo } \\
\text { De acuerdo } \\
\text { Totalmente de } \\
\text { acuerdo }\end{array}$ & 1 & 2,6 \\
\hline $\begin{array}{l}\text { La sensibilidad social, la cercanía con las } \\
\text { problemáticas del contexto político, influyen en } \\
\text { la elección }\end{array}$ & $\begin{array}{c}\text { Totalmente en } \\
\text { desacuerdo } \\
\text { Poco acuerdo }\end{array}$ & 14 & 36,8 \\
\hline & De acuerdo & 1 & 60,5 \\
\hline & Totalmente de \\
acuerdo & 6 & 23,6 \\
\hline
\end{tabular}

Para los líderes estudiantiles con cargos de representación es el carisma un don especial una de las principales cualidades para ser elegidos representantes, un $60.5 \%$ totalmente de acuerdo y un $36.8 \%$ de acuerdo. Mientras que no lo consideran tan importante un 2.6\%. La coherencia entre lo que se dice y lo que se hace, las acciones que respaldan las palabras, es considerada por los estudiantes líderes como un gran valor para ser elegido representante, totalmente de acuerdo un 76:3\% y de acuerdo un $23.7 \%$. Se valora en $100 \%$.

Ser una persona comprometida, responsable y entregada, es una cualidad muy importante para los líderes estudiantiles. Totalmente de acuerdo un $92.1 \%$, de acuerdo un 7,9\%. Se concluye que el liderazgo exige compromiso con los electores y con la institución, u organización. Por su parte, la fidelidad a su compromiso con los compañeros y con la institución se considera un valor muy importante para ser elegido como representante. Un $76.3 \%$, estuvo totalmente de acuerdo, $15,8 \%$ de acuerdo, y poco acuerdo $7,9 \%$. La honestidad como un valor ético, escaso especialmente en la vida política, es considerada por los estudiantes representantes como uno de los valores que deben caracterizar a los líderes. Un 92.1\% estuvo totalmente de acuerdo. Y un 7,9\% de acuerdo, lo que concluye que la honestidad es un valor determinante para ser considerado digno y de confianza para ser elegido por los compañeros como representante ante la instancia gubernamental de la institución universitaria.

Los estudiantes están totalmente de acuerdo en un $84.2 \%$ y un $15,8 \%$ de acuerdo, que la lealtad, como la transparencia y la franqueza frente a los compañeros y la institución, es una de las principales cualidades de un líder estudiantil., para merecer ser elegido ante el gobierno universitario. Los representantes estudiantiles consideran que es importante el desarrollo de la inteligencia interpersonal, tener habilidades sociales y políticas, tener la empatía para llegarle a todos, es condición para ser elegido. Totalmente de acuerdo un $47.4 \%$ y un $44.7 \%$ de acuerdo. Un $7,3 \%$ no lo considera tan 
importante. Los representantes estudiantiles consideran la experiencia en el liderazgo para ser elegidos, un 28,9\% si la consideran indispensable, un $44,7 \%$ importante, $18,4 \%$ no la consideran tan importante y un $7,9 \%$ no la consideran necesaria para asumir los cargos de representación.

Los estudiantes consideran, que la presentación personal es importante para la elección como representante, se mantiene equilibrio entre totalmente de acuerdo, 39.5\%, lo mismo que de acuerdo. En nuestra cultura universitaria, por ser la Institución de carácter privado se exige la buena presentación y el uso impecable del uniforme en las facultades de salud. Un $39,5 \%$ está de acuerdo y un $21,1 \%$ no la considera como indispensable para los cargos de representación.

La variable género no se considera esencial para la elección como representante de los estudiantes, el 36,8\% poco acuerdo y el $31.6 \%$ totalmente en desacuerdo. El 68\% no considera que el género influya en la elección como representantes. La condición humana de ser varón o mujer no influye en la elección como representantes estudiantiles.

Los estudiantes consideran que la simpatía es una característica esencial para la elección como representantes, un $50 \%$, totalmente de acuerdo y un 31.6\% de acuerdo. El mantener buenas relaciones compañeros, ser simpático, caer bien es clave para la elección como representante e influye en la elección. El 60.5\% de los representantes estudiantiles, está en total acuerdo de considerar el valor de la humildad como muy importante para ser elegido en los órganos de representación en la Universidad. Frente a un $23,7 \%$ que está de acuerdo y un 15,8\% que no la considera como importante para ser elegidos como representantes.

La sencillez, ser descomplicado es uno de los valores considerados como muy importantes para la elección como representantes estudiantiles. El $60,5 \%$ de los representantes está totalmente de acuerdo. Un 26,3\% de acuerdo y un 13,2\%, que no considera esta cualidad tan necesaria para ser elegido como representante.

En relación a si los candidatos han sido presentados por los profesores y directivos un $42,1 \%$ opina estar en poco acuerdo y un $23,7 \%$ en total desacuerdo, frente un 23,7\% totalmente de acuerdo y un 10,5\% de acuerdo. Los directivos y profesores respetan la independencia, la libertad y autonomía de los estudiantes en los procesos electorales.

Los representantes consideran que tener una personalidad fuerte de alguna manera influye en la elección para los órganos de representación, de acuerdo $60.5 \%$ y en total acuerdo $21.1 \%$. El 13,2\% manifiesta poco acuerdo $\mathrm{y}$ el 5,3\% en total desacuerdo. La franqueza como un rasgo de la personalidad, es considerada en un $63.2 \%$ y $34.2 \%$, como una cualidad muy importante para ser elegido como representante estudiantil. Mientras que un 2,6\% manifiesta poco acuerdo. 
Los representantes estudiantiles consideran que la sinceridad, ser claro y transparente. como rasgo de la personalidad es fundamental para la elección en los órganos del gobierno. Un 71, $1 \%$ total acuerdo y u $28.9 \%$ de acuerdo. Los estudiantes representantes consideran la firmeza como rasgo de la personalidad como una cualidad muy importante para ser elegidos en los órganos del gobierno. Un $73.7 \%$ en total acuerdo y un $23.7 \%$ de acuerdo. Lo que demuestra el valor de esta cualidad, clave para el ejercicio del liderazgo.

La capacidad para la toma de decisiones, como un rasgo de la personalidad, es considerada como una cualidad para el ejercicio del liderazgo en los cargos de representación estudiantil. Un 78.9 totalmente de acuerdo, un 15.8 de acuerdo. Un 5,3\% con poco acuerdo. El poseer las cualidades de personalidad fuerte, franqueza, sinceridad y decisión firme son valoradas como fundamentales para el ejercicio del liderazgo de representación. 6,5\% totalmente de acuerdo, 36,8\% de acuerdo.

Por último, los representes estudiantiles consideran que la sensibilidad social y la cercanía con las problemas del contexto social y político si influyen en su elección. 44,7\% totalmente de acuerdo y un 36\% de acuerdo. Aunque el 15,8\% con poco acuerdo y un 2,6\% totalmente en desacuerdo.

\section{Conclusion}

Las causas de sus elecciones son múltiples y variadas y obedecen factores internos como cualidades de liderazgo rasgos de su personalidad, tener carisma, ser sociables, extrovertidos, altruistas, con control emocional, sinceros, leales, honestos, transparentes, ... así como a habilidades y destrezas, seguridad, firmeza, experiencias, servicio externas, más que la excelencia académica, la presencia o el estrato social (Cáceres, 2007; Cáceres, Aznar y Raso, 2013; Cáceres, Sachicola e Hinojo, 2015).

De este modo, observamos como ciertas cualidades son más valoradas que otras en función de la investigación llevada a cabo, que ha demostrado las diferentes motivaciones que llevan al alumnado a elegir a sus representantes y líderes estudiantiles.

Así pues, se finaliza pensando que ciertas cualidades son más valoradas que otras para la consecución de objetivos comunes. El papel del líder estudiantil es de especial relevancia para la comunidad educativa, concretamente para la Universidad de Santo Tomás de Bucaramanga, foco donde se ha realizado el estudio de esta investigación (Jaime, Cáceres e Hinojo, 2016).

\section{References:}

1. Bass, B. M. (1985). Leadership and performance beyond expectation. New York: Free Press. 
2. Bennis, W.G. (1959). Leadership theory and administrative behavior: The problem of authority. Administrative Science Quarterly, 4, 259201.

3. Cáceres, Mª . P. (2007). El liderazgo estudiantil en la Universidad de Granada desde una perspectiva de Género (Tesis doctoral inédita). Universidad de Granada: Departamento de Didáctica y Organización Escolar. Facultad de Ciencias de la Educación.

4. Cáceres, Mª. P., Lorenzo, M., \& Sola, T. (2008). Evaluación de la representación estudiantil en la Universidad desde un enfoque de género: diseño de un cuestionario. Enseñanza \& Teaching. 26, 137164.

5. Cáceres, Mª. P., Trujillo, J.M., Hinojo, F.J., Aznar, I. y García, M. (2012). Tendencias actuales de género y liderazgo de la dirección en los diferentes niveles educativos. EDUCAR "El liderazgo en las organizaciones educativas. Nuevos avances”. Gener-juny,48,(1), 6989.

6. Cáceres, Mª. P., Aznar, I. y Raso, F. (2013). Perceptions of Student Leadership in the University Context. The case of Student's Union in the University of Sheffield (United Kingdom). Open Journal of Leadership, Vol. 2, n 3, 68-72. SciRes, Scientific Research Group. USA.

7. Cáceres, Mª P., Sachicola, A. e Hinojo, Ma . A. (2015). Análisis del liderazgo femenino y poder académico en el contexto universitario español. En European Scientific Journal (ESJ), Vol. 11, N ${ }^{\circ} 2296-$ 311.

8. Campbell, D. (1991). Tha challenge of assessing leadership characteristics. Leadership in Action,11(2), 1-8.

9. Fiedler, F.E. (1967). A Theory of Leadership Effectiveness. New York: McGraw-Hill.

10. Gento, S. (1996). Instituciones educativas para la calidad total. Madrid: La Muralla.

11. Ginnett, R. C. (1996). Team effectiveness leadership model: Identifying leverage points for change. En Proceedings of the 1996 National Leadership Institute Conference.

12. Hogan, R., Curphy, G. J., \& Hogan, J. (1994). What we know about leadership: Effectiveness and personality. American psychologist, 49(6), 493.

13. Jaime, Mª P., Cáceres, M. P., \& Hinojo, F. J. (2016). Analysis of leadership styles developed by teachers and administrators in technical-technological programs: the case of the Cooperative University of Colombia. International Journal of Leadership in Education, 1-16. 
14. López, M. (2016). Liderazgo estudiantil en la Universidad Santo Tomás de Bucaramanga. Propuesta pedagógica (Tesis doctoral inédita). Universidad de Granada: Departamento de Didáctica y Organización Escolar. Facultad de Ciencias de la Educación.

15. Lorenzo, M. (2005). El liderazgo en las organizaciones educativas: revisión y perspectivas actuales. Revista Española de Pedagogía, 232, 367-388.

16. Meindl, J. R., \& Ehrlich, S. B. (1987). The romance of leadership and the evaluation of organizational performance. Academy of Management Journal, 30, 91-109.

17. Merton, R.K. (1969). The social nature of leadership. American Journal Nurs. 69, 2614-2618.

18. Rauch, C. F., \& Behling, O. (1984). Functionalism: Basis for an alternate approach to the study of leadership. En J. G. Hunt, D. M. Hosking, C. A. Schriesheim, and R. Stewart (Eds.), Leaders and managers: International perspectives on managerial behavior and leadership. New York: Pergamon Press, pp. 45-62.

19. Tichy, N. M., \& Devanna, M. A. (1986). The transformational leader. New York: Wiley.

20. Vázquez, L. (2010). Habilidades directivas y técnicas de liderazgo. Bogotá: Ediciones de la U. 